\title{
1789 / 1917 : l'ambivalence du discours révolutionnaire des communistes français des années 1930
}

1789/1917 : the ambivalence of french communists' revolutionary speech in the thirties

1789/1917 : la ambivalencia del discurso revolucionario de los comunistas franceses de los años 1930

\section{Damon Mayaffre}

\section{OpenEdition}

Journals

Édition électronique

URL : https://journals.openedition.org/mots/10373

DOI : $10.4000 /$ mots. 10373

ISSN : 1960-6001

Éditeur

ENS Éditions

Édition imprimée

Date de publication : 1 juillet 2002

Pagination : 65-79

ISBN : 2-84788-011-9

ISSN : 0243-6450

\section{Référence électronique}

Damon Mayaffre, « 1789 / 1917 : l'ambivalence du discours révolutionnaire des communistes français des années 1930 », Mots. Les langages du politique [En ligne], 69 | 2002, mis en ligne le 13 mai 2008, consulté le 23 avril 2022. URL : http://journals.openedition.org/mots/10373 ; DOl : https://doi.org/ $10.4000 /$ mots. 10373 


\section{9 / 1917 : l'ambivalence du discours révolutionnaire des communistes français des années 1930}

Le surgissement du parler communiste dans la rhétorique politique française est depuis longtemps l'objet d'une attention soutenue ${ }^{1}$. Dans la logocratie parlementaire de la Troisième République, le discours communiste a résonné, tout de suite, d'une tonalité particulière.

Le souci identitaire et organisationnel par exemple (« Nous, les communistes, nous devons toujours mieux nous organiser... $\rangle^{2}$ ) le marque au fer rouge et, dès lors, le discours semble avant tout le lieu ou le moyen de l'établissement ou de l'étanchement de la "subsociété »" qu'historiens et sociologues ont reconnue, en France, dans la réalité communiste.

Pour autant il est exagéré de parler d'une idiomatique communiste propre: en dépit des affirmations d'Annie Kriegel, il est facile de constater qu'il ne s'agit ni d'un argot, ni d'un sous-langage codé, encore moins d'un « langage ésotérique ${ }^{4}$; pour finir rares sont les mots (social-fascisme, rayon, etc.) qui lui sont exclusifs.

Plus simplement il faut voir dans le discours communiste des origines - nos constats se borneront, ici, à l'entre-deux-guerres -, un discours politique atypique en ce sens qu'il a moins le souci de nourrir le débat logocratique, bourgeois et parlementaire de la République française que de rompre avec lui.

- CNRS-UMR 6039 Bases, Corpus et Langage, Faculté des Lettres, 98 bd. E Herriot BP 3209 - 06204 Nice cedex.

1. D. Labbé, 1977, Le discours communiste, Presse de la FNSP. D. Peschanski, 1989, Et pourtant ils tournent : vocabulaire et stratégie du PCF (1934-1936), INaLF, Klincksieck.

2. Phrase prototypique que l'on trouve par exemple au début des années 1930 sous la plume de M. Thorez dans les Cahiers du bolchevisme, 1, janvier 1930.

3. A. Kriegel, 1974, « Langage et stratégie », Communisme au miroir français, NRF, p. 98.

4. Ibid., p. 99 . 
En d'autres termes, le discours communiste peut être défini comme un discours révolutionnaire par deux de ses fonctions principales et originales qui tranchent avec les archétypes discursifs républicains que nous connaissons : organiser de manière obsessionnelle le Parti, bras armé de la révolution, et inciter, au quotidien, à l'action de rupture, à la lutte, au combat comme répétition générale du grand soir.

Ces affirmations demandent évidemment à être démontrées. La comparaison rigoureuse que l'on fera du discours de M. Thorez entre 1930 et 1939, non seulement avec le discours de droite (P.-E. Flandin et A. Tardieu) mais plus pertinemment avec le discours socialiste (L. Blum) nous apportera les preuves de la marginalité révolutionnaire du discours. Plus subtilement surtout, ces affirmations demandent à être nuancées de manière diachronique car l'on sait que le discours communiste connait dans les années 1930 un vif changement qui le mènera, cahin-caha, à sa forme consensuelle actuelle : et ce changement se fait précisément autour d'un glissement référentiel de l'acte de parole révolutionnaire défini non plus en rapport à 1917 mais en rapport à 1789 .

\section{Un discours révolutionnaire}

La comparaison systématique des discours de M. Thorez, secrétaire général du PCF, avec les discours de trois dirigeants politiques majeurs et représentatifs des principales autres forces politiques de l'époque (L. Blum pour les socialistes, P.-E. Flandin pour le centre-droit orléaniste, A. Tardieu pour la droite bonapartiste et autoritaire ${ }^{5}$ ) atteste de l'exceptionnalité du parler communiste dans le concert politique français de l'entre-deux-guerres. Il s'agit même là du tout premier constat $\mathrm{du}$ traitement lexicométrique du grand corpus de textes politiques de notre thèse, constitué de 1.600 .000 mots (400.000 par locuteurs) et 832 discours $^{6}$.

5. M. Thorez, L. Blum, P.-É. Flandin et A. Tardieu sont quatre personnages importants des années 1930. Tous quatre furent députés et leaders d'une mouvance politique influente. M. Thorez en tant que secrétaire collégial, puis secrétaire général du PCF incarne à lui seul, par le fait du centralisme démocratique, la parole officielle du parti communiste. L. Blum fut pendant tout l'entre-deux-guerres le leader de la SFIO, éditorialiste du Populaire, et président du groupe socialiste à l'Assemblée ; il fut 3 fois président et vice-président du Conseil entre 1936 et 1938. P.-É. Flandin fut le président de l'Alliance démocratique, premier parti de centre droit. Il fut 11 fois ministre et 1 fois président du Conseil. A. Tardieu enfin est le chef incontesté des droites au début des années 1930 ; il fut 6 fois ministre et 3 fois président du Conseil.

6. D. Mayaffre, 2000, Le poids des mots. Le discours de gauche et de droite dans l'entredeux-guerres. M. Thorez, L. Blum, P.-Et. Flandin et A. Tardieu (1928-1939), Honoré Champion, 


\section{Un vocabulaire révolutionnaire}

L'analyse des correspondances effectuée sur l'ensemble du vocabulaire des quatre dirigeants situe en effet sans ambigüité $\mathrm{M}$. Thorez en marge. Sur l'axe 1 (l'axe horizontal qui regroupe $96 \%$ de l'information), l'opposition est criante entre M. Thorez et les 3 autres orateurs (figure 1).

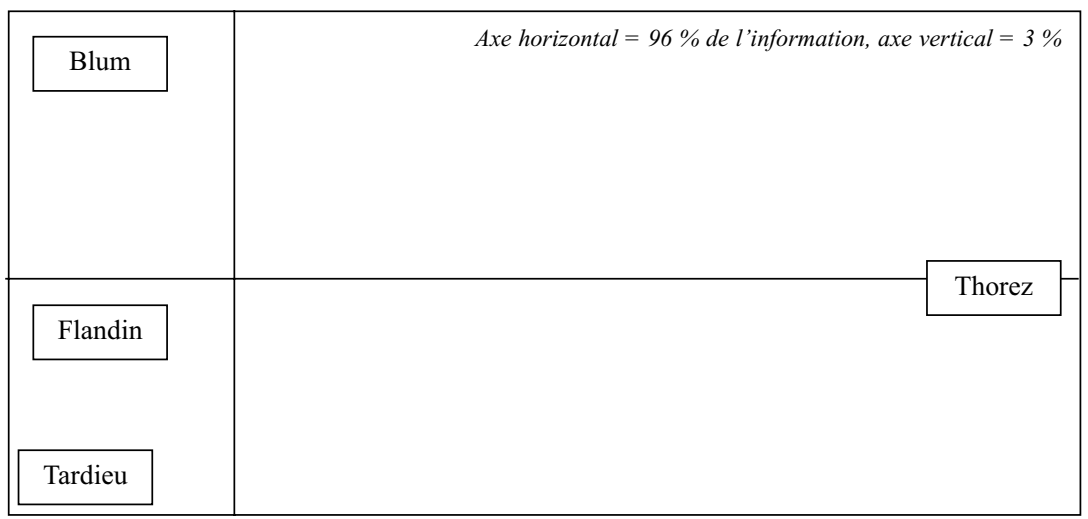

Figure 1 : Analyse factorielle du vocabulaire

Cela signifie que le véritable clivage discursif du monde politique français se fait non pas entre M. Thorez et L. Blum vs P.-É. Flandin et A. Tardieu comme on pouvait s'y attendre mais, violemment, entre le locuteur communiste et les autres locuteurs.

Les mots qui contribuent à cette distinction majeure ne sont pas difficiles à trouver lorsque l'on regarde la liste des spécificités du discours communiste que fournit le logiciel Hyperbase ${ }^{7}$.

Il s'agit avant tout de l'ensemble du vocabulaire de lutte, de combats, d'actions revendicatives ou subversives qualifiées le plus souvent par M. Thorez de révolutionnaires. Dans le corpus, lutte(s), grève(s), combat(s) ou l'adjectif révolutionnaire(s) par exemple appartiennent en

800 p. Le corpus de ce travail recueille l'exhaustivité des discours et articles de M. Thorez et une bonne partie de ceux de L. Blum, P.-É. Flandin et A. Tardieu entre 1928 et 1939. Il est, comme l'exigent les traitements lexicométriques, homogène et équilibré, contrastif et diachronique favorisant ainsi les comparaisons (p. 38-51).

7. Hyperbase est un des meilleurs logiciels de lexicométrie proposé actuellement sur le marché. Conçu par E. Brunet et l'UMR 6039 «Bases, corpus et langage» du CNRS (NiceS. Mellet), il est disponible sous Mac ou sous PC et est fourni avec un mode d'emploi. 
propre à $\mathrm{M}$. Thorez et se distribuent comme l'indique le graphique 1 entre les 4 locuteurs $^{8}$ :

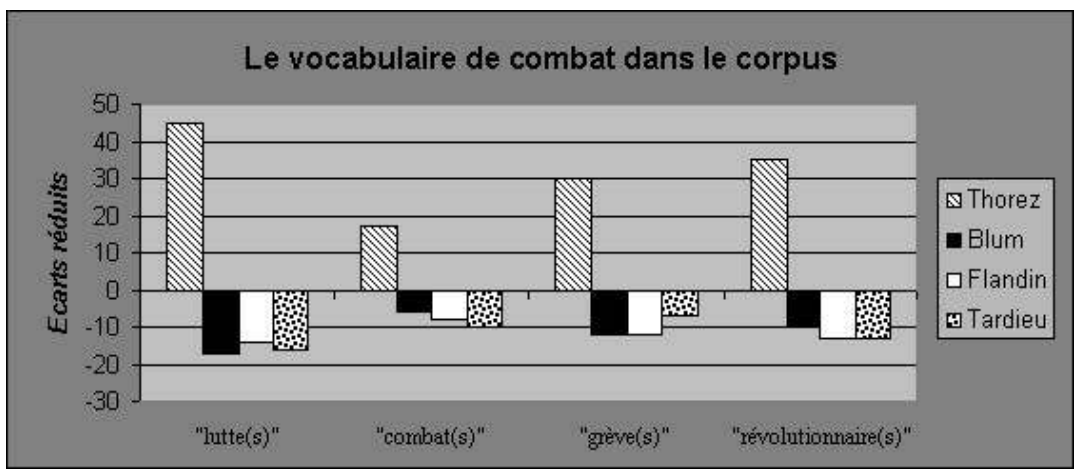

Graphique 1: Le vocabulaire de combat révolutionnaire dans le corpus : distribution politique

Si le vocabulaire discriminant du discours de M. Thorez est le vocabulaire de lutte, précisons qu'il s'agit avant tout du vocabulaire de lutte... des classes, c'est-à-dire au fond, de l'ensemble des mots marxistes signifiant la division bipolaire de la société en deux classes sociales qui ont vocation à s'affronter. Bourgeoisie et ouvrier(s), prolétariat ou classe sont en effet l'apanage du discours communiste (graphique 2) :

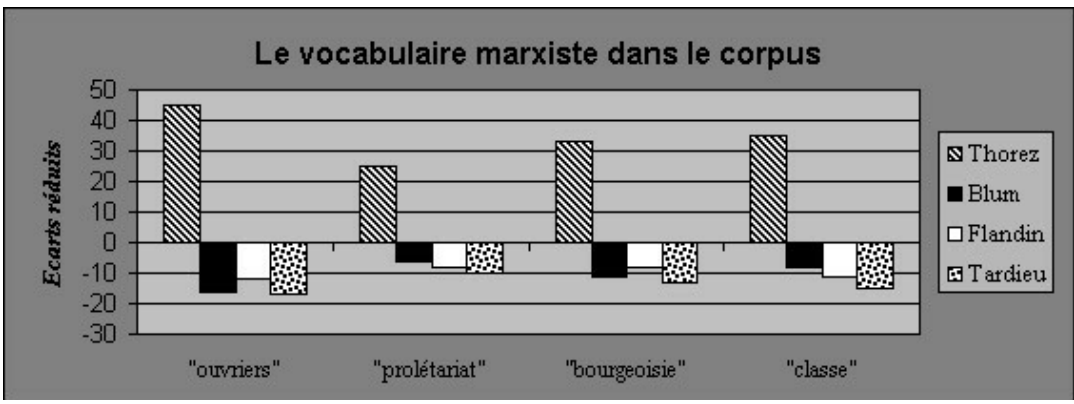

Graphique 2 : Le vocabulaire marxiste dans le corpus : distribution politique

8. La lecture du graphique suivant est très simple. L'écart réduit mesure le degré de sur-utilisation ou de sous-utilisation d'un mot par un locuteur par rapport à la norme du corpus. Si les 4 locuteurs utilisaient avec la même intensité lutte(s), il n'y aurait pas de différence entre eux : sur le graphique, les bâtonnets représentant les écarts réduits seraient égaux et proches de 0 ; ils ne se détacheraient pas de l'axe des abscisses. Ici au contraire on enregistre une sur-utilisation 
Ces constats sans appel possible ${ }^{9}$ nous intéressent au fond finalement autant pour M. Thorez que pour L. Blum. Le discours de M. Thorez est un discours qui met en scène un affrontement révolutionnaire entre classes sociales antagonistes ${ }^{10}$, lorsque le discours socialiste, déjà dans l'entre-deux-guerres, s'inscrit dans la tradition parlementaire mettant en scène le débat républicain. Chez L. Blum, à l'absence remarquable des mots du combat révolutionnaire répond la redondance de ceux de la délibération : débat, Chambre, motion, opinion, pensée, idée, contreprojet, hypothèse, conviction, etc. ${ }^{11}$. Et le discours de M. Thorez au regard non seulement du discours de droite mais du discours de L. Blum apparait bien comme atypique par son aspect combatif et subversif.

\section{Une rhétorique révolutionnaire}

Fondamentalement, c'est donc la substance lexicale révolutionnaire (i.e. combative) qui discrimine le discours de M. Thorez.

Pourtant, c'est une autre différence - discursive cette fois-ci - que les linguistes ont repérée pour souligner l'originalité de la prose communiste.

Lucille Courdesses a en effet montré comment $\mathrm{M}$. Thorez - premier véritable dirigeant communiste de l'histoire de France ${ }^{12}-$ a inventé une forme de rhétorique nouvelle qui rompt avec la rhétorique parlementaire ${ }^{13}$. Non sans jouer sur les mots, on peut affirmer que le discours de M. Thorez apparait ainsi révolutionnaire en ceci qu'il révolutionne la rhétorique politique traditionnelle.

En effet, là où les discours habituels se présentent, selon la typologie de Jean Dubois ${ }^{14}$, comme des discours polémiques fonctionnant notam-

massive de lutte(s) par M. Thorez (+45) «compensée» par une sous-utilisation du mot par L. Blum (-16), P.-É. Flandin (-12) et A. Tardieu (-17).

9. Nous n'insisterons pas ici sur l'importance statistique de la valeur des écarts réduits pour les mots ci-dessus traités.

10. On notera à ce propos l'extraordinaire sur-utilisation de la préposition contre $(+45)$ dans le discours communiste par rapport au discours politique moyen (D. Mayaffre, 2000, p. 139).

11. Pour une analyse du discours réformiste, parlementaire et « performatif » de L. Blum voir D. Mayaffre, 2000, p. 198-209.

12. Rappelons que M. Thorez, né en 1900, est le premier véritable leader d'un type nouveau emprunt de léninisme et de bolchevisme. Cachin, né en 1869, épouse la cause communiste dès le congrès de Tours mais il a été formé, avant-Guerre, à l'école politique et rhétorique de la Troisième République.

13. Pour l'ensemble du développement qui suit voir L. Courdesses, 1971, «Blum et Thorez en mai $1936 »$, Langue Française, 9, p. 22-34.

14. J. Dubois, 1969, «Énoncé et énonciation », Langages, 13, p. 100-110 et J. Dubois, 1969, « Lexicologie et analyse d'énoncé », Cahiers de lexicologie, 15, p. 119-121. 
ment sur une tension très forte entre le locuteur et l'auditeur (opposition je/vous), le discours communiste se présente comme un discours didactique qui sous-entend une communauté de pensée, déjà acquise dans l'énonciation, entre l'orateur et l'auditoire (double masquage du locuteur et de l'auditeur, et énonciation collective et universelle sur le mode d'un nous fédérateur voire phagocytaire).

Ainsi si l'on étudie le procès de l'énonciation des discours et la distribution des je et des vous dans le corpus, le résultat est remarquable par son aspect caricatural (graphique 3) :

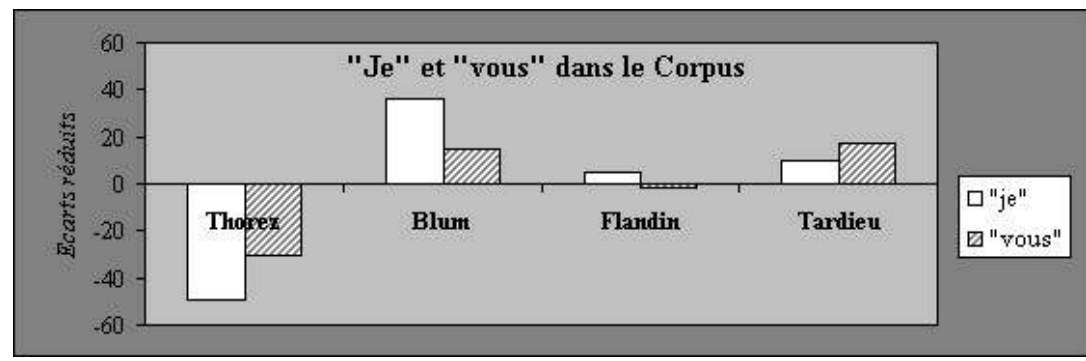

Graphique 3 : Je et vous dans le corpus : distribution politique

Je et vous sont en fait les deux mots, parmi tous, les plus sous-utilisés par M. Thorez ; très présents chez L. Blum, P.-É. Flandin et A. Tardieu, ils sont quasiment absents du discours de M. Thore ${ }^{15}$. Naturellement, cette sous-utilisation des je (et des me, mon, mien, etc.) par M. Thorez est compensée dans son discours par une sur-utilisation du nous $(+14)$ et de notre $(+35)$. À l'inverse de la première personne du singulier, les nous dans la prose communiste se multiplient à l'excès pour saturer le texte comme l'illustre, entre mille, le passage suivant :

Nous, Parti communiste, mettant au-dessus de tout notre volonté de nous opposer à la guerre, de nous opposer au gouvernement dit d'union nationale... nous voulons... ${ }^{16}$.

Ce discours didactique qui se décline sur le mode d'un nous exclusif de tout autre pronom personnel est donc frappant par sa particularité. En revanche, il n'est pas difficile de comprendre sa genèse; du reste, seules des causes profondes peuvent expliquer que cette rhétorique thorézienne

15. M. Thorez, dans l'ensemble de ses discours pour toutes les années 1930 prononce en moyenne seulement 35 fois je par an. Dans le même temps, sur un corpus de même taille et de même qualité, L. Blum en prononce 350 .

16. M. Thorez, Discours prononcé à la Chambre des députés, le 15 juin 1934 (Oe. MT, LII, t. 6, p. 124-129). 
soit définitivement adoptée par les dirigeants communistes jusqu'au tournant des années $19900^{17}$.

La négation de la personnalité du locuteur (bannissement du je) et celle de l'auditeur (bannissement de vous ou de $t u$ ) semble induite par l'organisation du Parti et le centralisme démocratique. Par les vertus de l'idéal démocratique, le secrétaire général se présente comme un simple porte-parole des militants qui ont fait remonter au sommet leurs exigences : M. Thorez ne saurait parler en son nom personnel mais se doit de parler au nom de tous les communistes («nous, les communistes, nous pensons... »). Surtout par le fait du centralisme, le PCF n'admet pas de contradiction interne. Le parti est un bloc et toute décision, toute parole - la ligne - doivent être admises par tous : le nous est alors le pronom qui représente ce bloc uni et unanime.

L'énonciation de M. Thorez apparait ainsi comme l'expression naturelle d'un parti bolchevique tel que le décrit Lénine dans Que faire? et l'impose par les 21 propositions. C'est non seulement l'expression d'un parti dans lequel l'individu s'abandonne pour porter haut un idéal collectif et collectiviste, mais l'expression d'un parti tout-un, d'un parti monolithique, sans contradiction et en ordre de marche pour la révolution.

$\mathrm{Au}$ final, le discours communiste apparait deux fois marginal dans le discours politique français ; en ce qu'il propose un modèle énonciatif original, en ce qu'il charrie une substance lexicale particulière.

Il est amusant de constater que ce double constat n'est pas sans présenter une contradiction peut-être fondamentale pour l'histoire du PCF.

Si l'affrontement lexical violent, dans l'énoncé, entre la bourgeoisie et les prolétaires semble inciter au combat révolutionnaire, la mise en scène, dans l'énonciation, de cet affrontement semble, elle, inciter à l'inertie. Les linguistes en effet insistent sur l'effet émollient du cadre énonciatif des discours didactiques : l'auditeur se trouvant déjà situé, dans l'instance du discours à l'intérieur d'un « nous-cocon », est rendu passif. Le didactisme par son énonciation pacifiée et universalisante (nous) aurait des vertus lénifiantes.

Alors, le discours communiste est-il un discours léniniste ou un discours lénifiant?

Au regard de l'échec historique du $\mathrm{PCF}$ de mener à bien la révolution en France, peut-être pensons-nous tenir une réponse.

17. La rhétorique thorézienne reste incontestablement très prégnante au moins jusqu'à George Marchais (D. Labbé). En revanche, elle semble abandonnée, sous réserve d'inventaire, par Robert Hue qui n'hésite pas à utiliser le je dans ses discours. 
Mais il reste que la prose communiste révolutionne les habitudes discursives parlementaires et en mettant frontalement en scène un monde bipolaire et manichéen, appelle plus à l'affrontement révolutionnaire qu'au consensus républicain.

\section{9 / 1917 : deux héritages lexicaux révolutionnaires concurrents ou complémentaires?}

Par sa substance lexicale avant tout, qui fait référence en des termes souvent martiaux à l'affrontement révolutionnaire, le discours communiste se différencie du discours politique moyen. Ce constat néanmoins demande à être affiné de manière diachronique puisque l'on sait que le « grand tournant» de 1933-1936 et la constitution du Front populaire font entrer le parti communiste dans une pratique réformiste du régime social et des institutions politiques (savoir arrêter une grève en juin 1936 lorsque des avancées sociales sont acquises ; sauver la république parlementaire menacée par le fascisme ; proposer la participation de ministres communistes au gouvernement bourgeois au début de 1938).

Dans un corpus diachronique (tous les discours de M. Thorez entre 1930 et 1939) partitionné annuellement et équilibré autour de la période du Front populaire, on enregistre en effet de profonds changements. Sans qu'il soit possible ici de tous les rappeler, il est à noter que le principal d'entre eux affecte les mots, les agents, les références à la révolution.

\section{Quelle révolution?}

À l'intérieur du corpus M. Thorez, les mots qui connaissent la distribution chronologique la plus spectaculaire au cours de cette décennie décisive que constituent les années 1930 sont en effet ceux qui désignent les acteurs révolutionnaires du discours.

Les ouvriers par exemple ou la classe ouvrière (plus généralement les prolétaires, le prolétariat, les travailleurs, etc. ${ }^{18}$ ) agents par définition de la révolution prolétarienne dans le discours communiste tendent à disparaitre.

18. Nous illustrerons notre propos ici uniquement par ouvrier(s) mais l'ensemble de la démonstration est valable pour tout le vocabulaire marxiste (prolétaires, prolétariat, travailleurs, etc., ou bourgeoisie, bourgeois, etc.). 
Utilisé en moyenne 70 fois tous les 10.000 mots par M. Thorez en 1930, ouvrier(s) n'est plus utilisé que 10 fois en 1939 (graphique 4) !

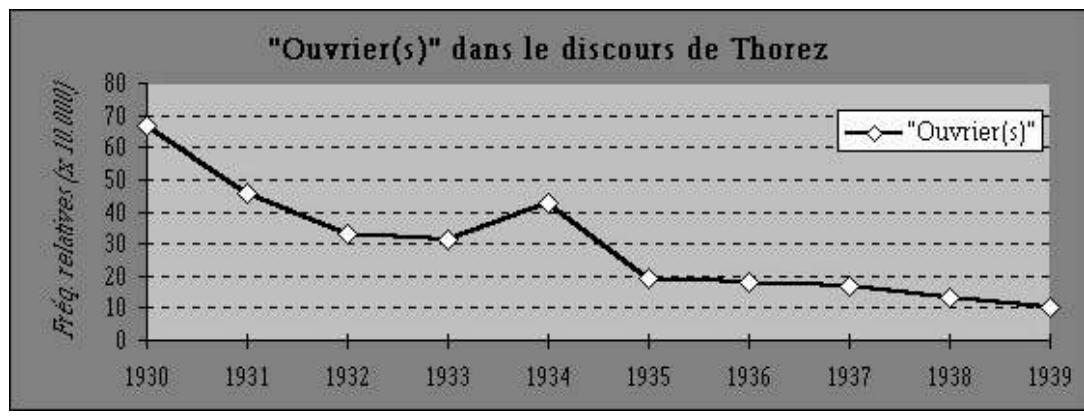

Graphique 4: Ouvrier(s) dans le discours de Thorez : distribution chronologique

Si les ouvriers tendent à disparaitre, qui devient l'acteur (révolutionnaire) du discours du PCF ? Qui remplace - car le discours ne saurait rester vide - la classe ouvrière, le prolétariat ou les travailleurs dans la prose communiste ? Hyperbase apporte sa réponse statistique et à la diminution des termes ouvriéristes correspond exactement une augmentation des termes populaires. Le mot peuple, totalement absent en 1930, rarissime en 1931, peu fréquent en 1932, envahit progressivement le discours pour être omniprésent entre 1936 et 1939 (graphique 5).

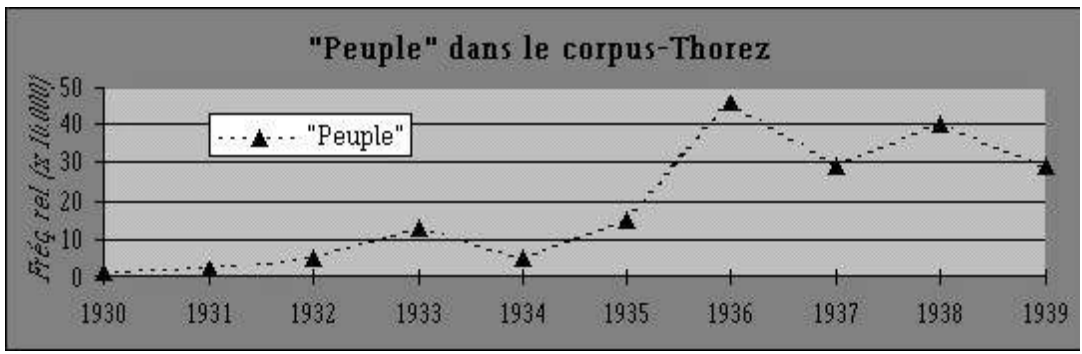

Graphique 5 : Peuple dans le discours de Thorez : distribution chronologique

Évidemment lorsque l'on superpose les deux derniers graphiques et que l'on calcule l'indice de corrélation des deux évolutions, on mesure combien les deux courbes sont symétriquement opposées et combien peuple chasse ouvriers du discours, à moins que cela soit ouvriers qui s'efface laissant ainsi place à peuple (graphique 6). 


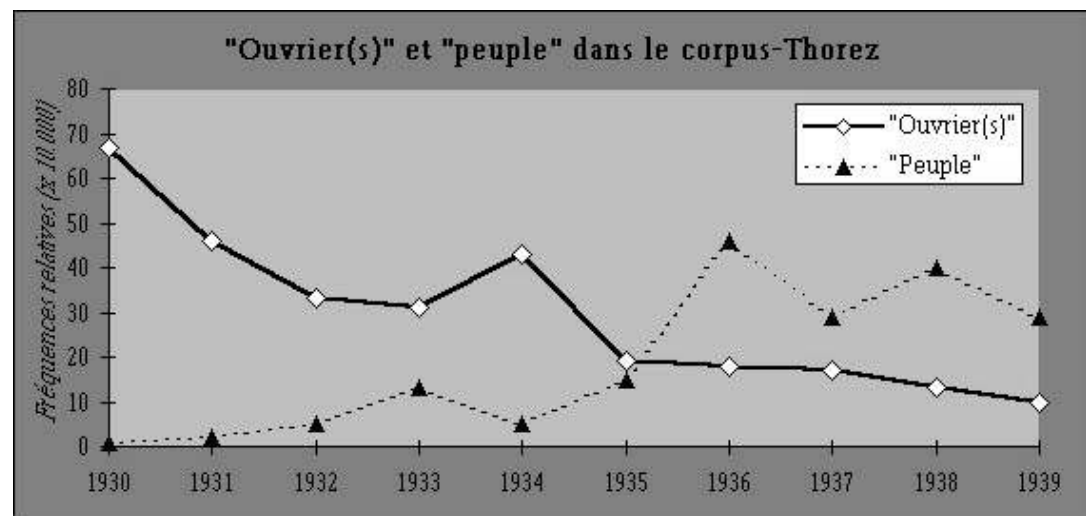

Graphique 6 : Ouvrier(s) et peuple dans le corpus-Thorez, distribution chronologique.

Avec le surgissement de peuple et l'effacement d'ouvriers ${ }^{19}$, c'est un glissement référentiel majeur de l'acte révolutionnaire qui s'opère dans le discours communiste. Peuple, sans être dépourvu d'une dimension sociale, est avant tout chargé d'une dimension politique et patriotique, en un mot jacobine ${ }^{20}$. Le terme masque l'opposition inter-classiste entre le travail et le capital, et n'a pas de résonance marxiste. Il fond - et a vocation à fondre dans le discours du Front populaire - dans la même entité la classe moyenne et les ouvriers, la petite bourgeoisie et le prolétariat, l'électorat du parti radical voire de la droite modérée et l'électorat du PCF, unis contre les seules 200 familles.

Ceci étant dit, peuple à lui tout seul réintroduit l'héritage révolutionnaire français dans le discours communiste. C'est la période en effet où M. Thorez se réapproprie l'histoire de la Révolution, réapprend la Marseillaise, se revendique des Jacobins :

C'est que nous sommes, nous communistes de France, les héritiers authentiques des jacobins de 1792. C'est que nous sommes les porteurs de l'héritage sacré de la Révolution comme des espérances les plus chères de notre peuple... ${ }^{21}$.

19. Le lecteur historien aura remarqué que ce surgissement et cet effacement se font de manière très précoce (1930-1933) et presque régulière. Seulement, 1934 revient en arrière et remet en cause la tendance générale au risque de nous cacher la précocité de l'évolution. C'est la datation du " grand tournant » du parti communiste - fixé trop tardivement et trop brutalement à 1935 - qui est ainsi à revoir (D. Mayaffre, 2000, p. 473-481).

20. Le terme peuple, sa signification, sa dénotation, sa connotation ont souvent été étudiés dans le discours politique français. On se réfèrera au plus récent ouvrage sur la question : Y. Meny et Y. Surel, 2000, Par le peuple et pour le peuple, Fayard, chap. 4, p. 182-225.

21. M. Thorez, Discours prononcé à l'occasion du centenaire de Rouget de Lisle, le 27 juin 1936. 
Sans aucun doute, avec cette revendication nouvelle mais constamment répétée, le discours communiste gagne un souffle révolutionnaire nouveau; mais il perd en même temps l'identité bolchevique qu'il s'était appliqué à se construire dans les années 1920.

Bien que M. Thorez s'en défende en prétendant établir un pont historique entre la Grande Révolution et la révolution russe, ou entre 1792 et $1936^{22}$, la référence à la Révolution bourgeoise de 1789 gomme progressivement dans la prose communiste la référence à la révolution prolétarienne de 1917. Comme illustré avec le chassé-croisé d'ouvrier(s) et de peuple, c'est l'ensemble du vocabulaire humaniste hérité des Lumières (hommes, enfants, démocratie, liberté) qui prend la place du vocabulaire marxiste (prolétaires, dictature du prolétariat, bourgeoisie, etc. ${ }^{23}$ ).

Plus simplement mais de manière significative, à partir du Front populaire, les occurrences des dates de la Révolution $(1789,1790$, etc.) remplacent celles de la révolution bolchevique. Dans des sous-corpus de même taille, M. Thorez fait allusion entre 1930 et 1935 seulement deux fois à 1789-1793, après 1935 et jusqu'à la veille de la guerre, il y fait allusion 52 fois. En proportion renversée M. Thorez fait référence 17 fois à 1917 avant 1935,3 fois après cette date.

\section{Renoncement révolutionnaire}

Évidemment, la référence à une révolution (bourgeoise) déjà réalisée et vieille de 150 ans pose la question de l'effectivité du message révolutionnaire du discours communiste dans la seconde partie des années 1930. Et s'il suffisait de se référer à 1789 pour donner à son discours des accents révolutionnaires, les discours de L. Blum ou des radicaux-socialistes apparaitraient presque aussi avancés que celui des communistes.

En fait, d'une manière plus générale, il faut conclure que l'appel à l'acte subversif révolutionnaire diminue dans les discours du fils du

22. C'est l'époque où $M$. Thorez en effet cultive assidument le parallèle entre la France de 1792 assiégée par la Prusse et les émigrés de Coblence, et la France de 1936 menacée par Hitler et trahie par les 200 familles et les chefs des ligues d'extrême[s] droite[s] « ces dignes continuateurs des émigrés de Coblence, ces nobles féodaux dont l'arrière-grand-père du comte de La Rocque, qui allaient mettre leur épée au service du roi de Prusse contre notre peuple fondant la Première République » (M. Thorez, 11 octobre 1936). La fuite des capitaux au moment du gouvernement Blum, la guerre d'Espagne ou Munich sont ainsi vécus comme un «nouveau Coblence », un « Coblence moderne » (M. Thorez, 5 avril et 6 aout 1936).

23. Pour les données chiffrées de ces constats lexicométriques voir D. Mayaffre, 2000, p. 416, 424 et $472-473$. 
peuple. Si l'on reprend la distribution de grèves, lutte... etc., qui distinguent tant le discours communiste du discours politique moyen, le constat est frappant (graphique 7) :

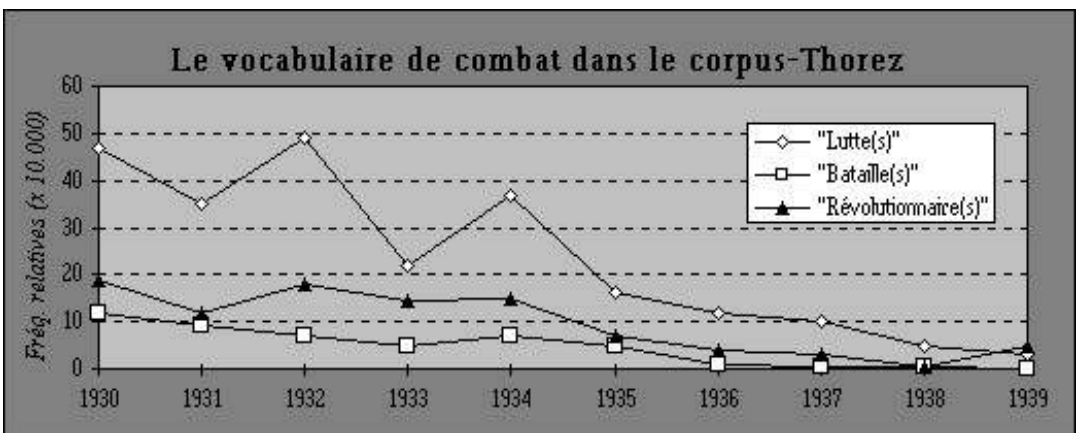

Graphique 7 : Le vocabulaire de combat dans le corpus-Thorez, distribution chronologique.

De la même manière grèves tend à disparaitre et il est par exemple extraordinaire de constater que le mot est quatre fois moins utilisé en 1936 - l'année du plus grand conflit social français du $20^{\mathrm{e}}$ siècle - qu'en 1930.

Le discours communiste dans l'entre-deux-guerres est un discours révolutionnaire qui perd donc, au fil des années, ses caractéristiques combatives voire seulement revendicatives. À la diminution de grèves, lutte ou combat s'ajoute en effet la quasi-disparition des mots pourtant presque anodins revendication(s) ou revendicatives. Des verbes ou des noms symptomatiques de la phraséologie communiste tendent aussi à diminuer statistiquement de manière irrésistible : combattre, arracher, combativité, agitation, attaque ${ }^{24}$.

Au final, même si, à partir de 1935, les références répétées à 1789 permettent de masquer l'évolution réformiste (i.e. non-subversive) du discours, elles en précipitent en fait l'évolution : si M. Thorez peut encore faire référence à la révolution, il renonce au grand soir prolétarien et s'inscrit désormais face à la menace fasciste et hitlérienne dans la préservation des acquis bourgeois de 1789, la République, la Nation, la démocratie, etc. ${ }^{25}$

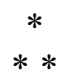

24. Pour les données chiffrées de ces constats lexicométriques voir D. Mayaffre, 2000, p. $422-423$.

25. Autant de mots quasi absents du discours de M. Thorez jusqu'en 1933-1935 et qui envahissent le discours avec le « grand tournant» (D. Mayaffre, 2000, p. 424 et 433). 
L'étude comparative de différents discours de l'entre-deux-guerres atteste de l'étrangeté de la parole communiste dans la polyphonie politique française.

Cette étrangeté tient-elle à l'aspect révolutionnaire du discours ? Pour répondre définitivement à cette question, sans doute faudrait-il définir les critères précis d'une prose révolutionnaire.

Si dans une définition ontologique le discours révolutionnaire est un discours qui appelle à l'action révolutionnaire, alors le discours de $\mathrm{M}$. Thorez en fait partie. Contrairement à l'usage classique, le discours de M. Thorez n'est pas un discours logocratique, centré sur lui-même, mais un discours de l'action voire du combat pour transformer le monde. Le discours politique de L. Blum par exemple se présente seulement - mais c'est déjà beaucoup - comme un " acte de discours » dans lequel se multiplient les verbes performatifs ou réflexifs sur le discours lui-même ( « je déclare que...», " je dis que... », « je répète que...») comme si l'essence même de l'action politique pour le leader socialiste était avant tout de penser, de parler, de délibérer. A contrario, le discours de M. Thorez est un appel à la mobilisation physique et au combat effectif. En termes austiniens, le discours politique parlementaire se singularise surtout par sa dimension illocutoire, lorsque le discours de M. Thorez a aussi une forte prétention perlocutoire. Et la perlocution que le discours communiste entend produire (se mettre en grève par exemple) espère avoir une dimension révolutionnaire.

Si un discours révolutionnaire est un discours qui fait référence à la révolution, là encore le discours de $\mathrm{M}$. Thorez répond au critère.

Pourtant, c'est ici que l'analyse se complexifie car dans une chronologie serrée on perçoit, même derrière certaines constances lexicales, un glissement du référent révolution de 1917 vers 1789. Surtout, l'évolution du vocabulaire au fil des années 1930 enregistre une diminution spectaculaire non seulement du vocabulaire marxiste mais du vocabulaire du combat au profit d'un vocabulaire humaniste et républicain.

Spécimen révolutionnaire dans le concert politique français, le discours communiste met donc en sourdine ses accents les plus remarquables, pour essayer à la veille de la guerre de se fondre dans une rhétorique jacobine plus traditionnelle.

Sans doute le rôle historique de M. Thorez fut-il de doter le parti communiste français d'une double culture : russe et française. Fervent lecteur de Lénine, soldat discipliné de la $\mathrm{III}^{\mathrm{e}}$ internationale, M. Thorez fut en même temps un grand admirateur de l'histoire populaire française qui vibrait à l'évocation des révolutions françaises $(1789,1848,1871)$. 
Très clairement, le Front populaire fut la période qui vit le PCF se réinscrire dans le paysage français lorsque le parti avait cultivé dans les années 1920 ses origines étrangères. Et explicitement, dans le corpus, $M$. Thorez en appelle durant cette période clef à une synthèse des deux traditions bolchevique et jacobine.

Pourtant, malgré les efforts du dirigeant communiste, cette synthèse n'est pas encore digérée avant la guerre - le sera-t-elle après la Résistance et la Libération? Ni synthèse, ni cohabitation, les deux références révolutionnaires légitimantes du discours des communistes français (1917 et 1789) s'entrechoquent encore dans les années 1930 et ne cohabitent que l'espace de quelques années charnières (1933-1935). Audelà, c'est en termes de concurrence et de succession que le lexique prolétarien bolchevique et le lexique humaniste français s'articulent dans le discours. Et dans les circonstances particulières du combat antifasciste de l'avant-guerre, le second semble triompher du premier. 